\title{
STABILITY OF SYZYGY BUNDLES ON AN ALGEBRAIC SURFACE
}

\author{
LaWrence Ein, Robert Lazarsfeld and Yusuf Mustopa
}

\begin{abstract}
We establish the stability of the syzygy bundle associated to any sufficiently positive embedding of an algebraic surface.
\end{abstract}

\section{Introduction}

The purpose of this paper is to prove the stability of the syzygy bundle associated to any sufficiently positive embedding of an algebraic surface.

Let $X$ be a smooth projective algebraic variety over an algebraically closed field $k$, and let $L$ be a very ample line bundle on $X$. The syzygy (or kernel) bundle $M_{L}$ associated to $L$ is by definition the kernel of the evaluation map

$$
\operatorname{eval}_{L}: H^{0}(L) \otimes_{k} \mathcal{O}_{X} \longrightarrow L .
$$

Thus $M_{L}$ sits in an exact sequence

$$
0 \longrightarrow M_{L} \longrightarrow H^{0}(L) \otimes_{k} \mathcal{O}_{X} \longrightarrow L \longrightarrow 0 .
$$

These vector bundles (and some analogues) arise in a variety of geometric and algebraic problems, ranging from the syzygies of $X$ to questions of tight closure. Consequently, there has been considerable interest in trying to establish the stability of $M_{L}$ in various settings. When $X$ is a smooth curve of genus $g \geq 1$, the situation is well-understood thanks to the work of several authors $[1,3,4,8,12,13]$; in particular, $M_{L}$ is stable as soon as $\operatorname{deg} L \geq 2 g+1$. When $X=\mathbf{P}^{n}$ and $L=\mathcal{O}_{\mathbf{P}^{n}}(d)$, the stability of $M_{L}$ was established by Flenner [9, Cor. 2.2] in characteristic 0 and by Trivedi [14] in characteristic $>0$ for many $d$. A more general statement, due to Coandǎ $[6]$, treats the bundles associated to possibly incomplete linear subseries of $H^{0}\left(\mathbf{P}^{n}, \mathcal{O}_{\mathbf{P}^{n}}(d)\right)$. Motivated by questions of tight closure, the stability of syzygy bundles on $\mathbf{P}^{n}$ arising from a somewhat more general construction has been studied by Brenner [2] and by Costa, Marques and Miró-Roig [7, 11]. In dimension 2, Camere [5] recently proved that kernel bundles on $K 3$ and abelian surfaces are stable.

We show here that if $L$ is a sufficiently positive divisor on any smooth projective surface $X$, then $M_{L}$ is stable with respect to a suitable hyperplane section of $X$. Specifically, fix an ample divisor $A$ and an arbitrary divisor $P$ on $X$. Given a large integer $d$, set

$$
L_{d}=d A+P
$$

and write $M_{d}=M_{L_{d}}$. Our main result is

Theorem A. If $d$ is sufficiently large, then $M_{d}$ is slope stable with respect to $A$.

Received by the editors November 28, 2012. 
Recall that the conclusion means that if $F \subseteq M_{d}$ is a subsheaf with $0<\operatorname{rank}(F)<$ $\operatorname{rank}\left(M_{d}\right)$, then

$$
\frac{c_{1}(F) \cdot A}{\operatorname{rank} F}<\frac{c_{1}\left(M_{d}\right) \cdot A}{\operatorname{rank} M_{d}} .
$$

Since a slope-stable bundle is also Gieseker stable, it follows that $M_{d}$ is parameterized for $d \gg 0$ by a point on the moduli space of bundles on $X$ with suitable numerical invariants. On the other hand, working over C, Camere [5, Proposition 2] shows that if $H^{1}\left(X, \mathcal{O}_{X}\right)=0$, and if the natural map

$$
H^{0}\left(X, K_{X}\right) \otimes H^{0}(X, L) \longrightarrow H^{0}\left(X, K_{X}+L\right)
$$

is surjective for some very ample line bundle $L$, then $M_{L}$ is rigid. However, this surjectivity is automatic if $K_{X}$ is globally generated and $L$ is sufficiently positive. Hence, we deduce

Corollary B. Let $X$ be a complex projective surface with vanishing irregularity $q(X)=0$, and assume that $K_{X}$ is globally generated. Then $M_{d}$ corresponds to an isolated point of the moduli space of stable vector bundles on $X$ when $d \gg 0$.

It is natural to suppose that the analogue of Theorem A holds also for varieties of dimension $\geq 3$, but unfortunately our proof does not work in this setting. However, if $\operatorname{Pic}(X) \cong \mathbf{Z}$, then the argument of Coandǎ [6] goes through with little change to establish:

Proposition C. Assume that $\operatorname{dim} X \geq 2$ and that $\operatorname{Pic}(X)=\mathbf{Z} \cdot[A]$ for some ample divisor A. Write $L_{d}=d A$. Then $M_{d}={ }_{\operatorname{def}} M_{L_{d}}$ is A-stable for $d \gg 0$.

As in [5] the strategy for Theorem $\mathrm{A}$ is to reduce the question to the stability of syzygy bundles on curves, but we avoid the detailed calculations appearing in that paper. In order to explain the idea, we sketch a quick proof of Camere's result [5, Theorem 1] that if $L$ is a globally generated ample line bundle on a K3 surface $X$, then $M_{L}$ is $L$-stable. Supposing to the contrary, let $F \subseteq M_{L}$ be a saturated destabilizing subsheaf, and fix a general point $x \in X$. Consider now a general curve $C \in\left|L \otimes \mathfrak{m}_{x}\right|$; we may suppose that $F$ sits as a sub-bundle of $M_{L}$ along $C$. Restriction to $C$ yields a diagram:

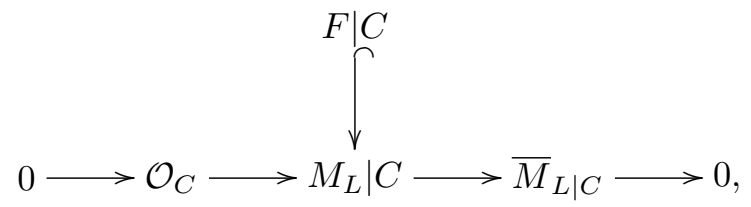

where $\bar{M}_{L \mid C}$ is the syzygy bundle on $C$ associated to $\Omega_{C}=L \mid C$. However, $\bar{M}_{L \mid C}$ is semi-stable by [13], while

$$
\mu(F \mid C) \geq \mu\left(M_{L} \mid C\right)>\mu\left(\bar{M}_{L \mid C}\right) .
$$

It follows that $F \mid C$ cannot inject into $\bar{M}_{L \mid C}$, and hence the two sub-bundles $\left.F\right|_{C}$ and $\mathcal{O}_{C}$ of $M_{L} \mid C$ have a non-trivial intersection, which in turn implies that $\mathcal{O}_{C}$ is contained in $\left.F\right|_{C}$. On the other hand, consider the fibres at $x$ of the various bundles in play. The vertical map in $\left(^{*}\right)$ corresponds to a fixed subspace $F(x) \varsubsetneqq H^{0}\left(X, L \otimes \mathfrak{m}_{x}\right)$. So we would be asserting that the equation defining a general curve $C \in\left|L \otimes \mathfrak{m}_{x}\right|$ 
lies in this subspace, and this is certainly not the case. The proof of Theorem A in general proceeds in an analogous manner, the main complication being that we have to deal with a trivial vector bundle of higher rank appearing on the left in the bottom row of $(*)$.

Concerning the organization of the paper, Section 1 is devoted to the proof of Theorem A. Proposition C appears in Section 2, where we also propose some open problems.

\section{Proof of main theorem}

This section is devoted to the proof of Theorem A from the Introduction.

We start by fixing notation and set-up. As in the Introduction, $X$ is a smooth projective surface, and $L_{d}=d A+P$ where $A$ is an ample divisor, and $P$ is an arbitrary divisor on $X$. For the duration of the argument, we fix an integer $b \gg 0$ such that $\left(b A+P-K_{X}\right)$ is very ample, and so that $H^{1}\left(X, L_{b}\right)=0$, and put

$$
B=L_{b}=b A+P .
$$

Observe that $b$ and $B$ are independent of $d$. We also assume henceforth that $d$ is sufficiently large, so that it satisfies the following properties:

(i) $L_{d}$ and $L_{d-b}$ are very ample.

(ii) For all $x \in X$, the natural mapping

$$
H^{0}\left(X,(d-b) A \otimes \mathfrak{m}_{x}\right) \otimes H^{0}(X, B) \longrightarrow H^{0}\left(X, L_{d} \otimes \mathfrak{m}_{x}\right)
$$

is surjective (where $\mathfrak{m}_{x}$ denotes the ideal sheaf of $x$ ).

Fixing such an integer $d$, assume now that $M_{d}=M_{L_{d}}$ is not $A$-stable. Recall that this means that there exists a non-trivial subsheaf

$$
F_{d} \subseteq M_{d}
$$

such that

$$
\frac{c_{1}\left(F_{d}\right) \cdot A}{\operatorname{rank} F_{d}} \geq \frac{c_{1}\left(M_{d}\right) \cdot A}{\operatorname{rank} M_{d}} .
$$

Without loss of generality, we assume that $F_{d} \subseteq M_{d}$ is saturated, and we fix a point $x=x_{d} \in X$ at which $F_{d}$ is locally free.

The plan is to use the stability of syzygy bundles on curves to show that if $d \gg 0$, then no such $F_{d}$ can actually exist. To this end, consider a general curve

$$
C_{d} \in|(d-b) A|=\left|L_{d}-B\right|
$$

passing through the fixed point $x \in X$. We may assume that $C_{d}$ is smooth and irreducible, and that $M_{d} / F_{d}$ is locally free along $C_{d}$. Observe also that for any torsionfree sheaf $\mathcal{F}$ on $X$ that is locally free along $C_{d}$, one has

$$
\mu_{A}(\mathcal{F})=\frac{1}{(d-b)} \cdot \mu\left(\mathcal{F} \mid C_{d}\right)
$$

In particular, if $\mathcal{F}$ is $A$-unstable as a sheaf on $X$, then $\mathcal{F} \mid C_{d}$ is unstable on $C_{d}$.

We now consider the restriction of $M_{d}$ and $F_{d}$ to $C_{d}$. Writing $\bar{M}_{d}=M_{\bar{L}_{d}}$ for the syzygy bundle on $C_{d}$ of the restriction $\bar{L}_{d}=L_{d} \mid C_{d}$, a straightforward analysis of the exact sequence $0 \rightarrow B \rightarrow L_{d} \rightarrow \bar{L}_{d} \rightarrow 0$ yields an exact sequence

$$
0 \longrightarrow H^{0}(B)_{C_{d}} \longrightarrow M_{d} \mid C_{d} \longrightarrow \bar{M}_{d} \longrightarrow 0,
$$


where the term on the left is the trivial bundle on $C_{d}$ with fibre $H^{0}(X, B)$. We complete this to a diagram

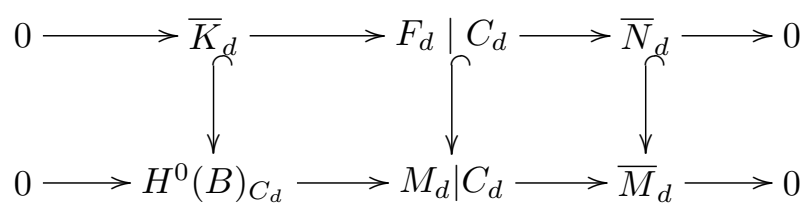

of vector bundles on $C_{d}$, where $\bar{N}_{d}$ denotes the image of $F_{d} \mid C_{d}$ in $\bar{M}_{d}$, and $\bar{K}_{d}$ is the kernel of the resulting map $F_{d} \mid C_{d} \longrightarrow \bar{N}_{d}$.

Observe now that

$$
L_{d}\left|C_{d} \equiv_{\operatorname{lin}}\left(C_{d}+B\right)\right| C_{d} \equiv_{\operatorname{lin}}\left(K_{X}+C_{d}+Q\right) \mid C_{d}
$$

where $Q=b A+P-K_{X}$ is very ample by assumption. In particular, $\operatorname{deg}\left(L_{d} \mid C_{d}\right)>$ $2 g\left(C_{d}\right)+1$, and hence $\bar{M}_{d}$ is stable on $C_{d}$ thanks to [8]. On the other hand, it follows from the bottom row of $(1.2)$ that $\mu\left(M_{d} \mid C_{d}\right)>\mu\left(\bar{M}_{d}\right)$, and hence

$$
\mu\left(F_{d} \mid C_{d}\right) \geq \mu\left(M_{d} \mid C_{d}\right)>\mu\left(\bar{M}_{d}\right) .
$$

Therefore $F_{d} \mid C_{d}$ cannot be a subsheaf of $\bar{M}_{d}$, and hence $\bar{K}_{d} \neq 0$.

The following two lemmas constitute the heart of the proof. The first asserts that the destabilizing subsheaf $F_{d}$ must have large rank.

Lemma 1.1. One has

$$
\operatorname{rank}\left(F_{d}\right) \geq h^{0}\left(\left(L_{d}-B\right) \otimes \mathfrak{m}_{x}\right)=h^{0}\left(L_{d}-B\right)-1 .
$$

The second lemma shows that if $d$ is sufficiently large, then the vertical inclusion on the left of (1.2) is the identity.

Lemma 1.2. If $d \gg 0$, then $\bar{K}_{d}=H^{0}(B)_{C_{d}}$.

Granting these assertions for now, we give the

Proof of Theorem A. We need to show that if $d \gg 0$ then the picture introduced above cannot occur. To this end, we consider the fibres at the fixed point $x \in X$ of the vector bundles appearing in the left hand square of (1.2). Since the fibre of the map eval ${ }_{L}$ at $x$ is the natural surjection $H^{0}\left(L_{d}\right) \rightarrow H^{0}\left(L_{d} \otimes \mathcal{O}_{x}\right)$, the fibre $M_{d}(x)$ of $M_{d}$ at $x$ is canonically identified with $H^{0}\left(X, L_{d} \otimes \mathfrak{m}_{x}\right)$, so these take the form

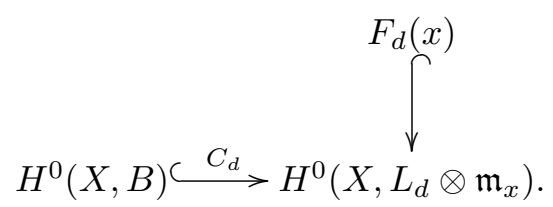

Here the bottom map is the natural inclusion determined by a local equation for $C_{d} \in\left|\left(L_{d}-B\right) \otimes \mathfrak{m}_{x}\right|$. It follows from Lemma 1.2 that $H^{0}(X, B)$ maps into the subspace

$$
F_{d}(x) \varsubsetneqq H^{0}\left(X, L_{d} \otimes \mathfrak{m}_{x}\right) .
$$

So for the required contradiction, it is enough to show that as $C_{d}$ varies over an open subset of $\left|\left(L_{d}-B\right) \otimes \mathfrak{m}_{x}\right|$, the images of the corresponding embeddings of $H^{0}(X, B)$ span all of $H^{0}\left(X, L_{d} \otimes \mathfrak{m}_{x}\right)$. But this follows from the surjectivity of the map (1.1). 
Proof of Lemma 1.1. We continue to work with the diagram (1.4), and we write $\mathbf{P}_{\text {sub }}(W)$ for the projective space of one-dimensional subspaces of a vector space $W$. Multiplication of sections gives rise to a finite morphism:

$$
\mu_{d}: \mathbf{P}_{\mathrm{sub}}\left(H^{0}\left(X,\left(L_{d}-B\right) \otimes \mathfrak{m}_{x}\right)\right) \times \mathbf{P}_{\mathrm{sub}}\left(H^{0}(X, B)\right) \longrightarrow \mathbf{P}_{\mathrm{sub}}\left(H^{0}\left(X, L_{d} \otimes \mathfrak{m}_{x}\right)\right) .
$$

Set

$$
Z=\mu_{d}^{-1}\left(\mathbf{P}_{\mathrm{sub}}\left(F_{d}(x)\right)\right)
$$

Then

$$
\operatorname{dim} \mathbf{P}_{\mathrm{sub}}\left(F_{d}(x)\right) \geq \operatorname{dim} Z
$$

thanks to the finiteness of $\mu_{d}$. On the other hand, for general $C_{d} \in\left|\left(L_{d}-B\right) \otimes \mathfrak{m}_{x}\right|$, the image of the corresponding inclusion

$$
H^{0}(X, B) \subseteq H^{0}\left(X, L_{d} \otimes \mathfrak{m}_{x}\right)
$$

must meet the subspace $F_{d}(x) \subseteq H^{0}\left(X, L_{d} \otimes \mathfrak{m}_{x}\right)$ non-trivially: indeed, this follows from (1.2) and the fact that $\overline{K_{d}}(x) \neq 0$. However, this means that the projection

$$
\operatorname{pr}_{2}: Z \longrightarrow \mathbf{P}_{\text {sub }}\left(H^{0}\left(X,\left(L_{d}-B\right) \otimes \mathfrak{m}_{x}\right)\right)
$$

is dominant. The Lemma follows upon combining $(*)$ and $\left({ }^{* *}\right)$.

Proof of Lemma 1.2. Since $M_{d} / F_{d}$ is locally free along $C_{d}$, it follows from (1.2) that $\bar{K}_{d}$ is a saturated subsheaf of $H^{0}(B)_{C_{d}}$, so it suffices to show that rank $\bar{K}_{d}=h^{0}(B)$. The argument is numerical. First, note from (1.2) and the stability of $\bar{M}_{d}$ that

$$
\begin{aligned}
\mu\left(F_{d} \mid C_{d}\right)=\frac{\operatorname{deg} \bar{K}_{d}+\operatorname{deg} \bar{N}_{d}}{\operatorname{rank} F_{d}} & \leq \frac{\operatorname{deg} \bar{N}_{d}}{\operatorname{rank} F_{d}} \\
& =\mu\left(\bar{N}_{d}\right) \cdot\left(\frac{\operatorname{rank} \bar{N}_{d}}{\operatorname{rank} F_{d}}\right) \\
& <\mu\left(\bar{M}_{d}\right) \cdot\left(1-\frac{\operatorname{rank} \bar{K}_{d}}{\operatorname{rank} F_{d}}\right) .
\end{aligned}
$$

Now $\operatorname{deg}\left(M_{d} \mid C_{d}\right)=\operatorname{deg}\left(\bar{M}_{d}\right)$, and since

$$
\mu\left(M_{d} \mid C_{d}\right) \leq \mu\left(F_{d} \mid C_{d}\right)
$$

equation (1.5) yields:

$$
\frac{\operatorname{deg}\left(M_{d} \mid C_{d}\right)}{\operatorname{rank} \bar{M}_{d}+h^{0}(B)}<\frac{\operatorname{deg}\left(M_{d} \mid C_{d}\right)}{\operatorname{rank} \overline{M_{d}}} \cdot\left(1-\frac{\operatorname{rank} \bar{K}_{d}}{\operatorname{rank} F_{d}}\right) .
$$

Observing that $\operatorname{deg}\left(M_{d} \mid C_{d}\right)<0$, this is equivalent to the inequality

i.e.,

$$
\frac{1}{\operatorname{rank} \bar{M}_{d}+h^{0}(B)}>\frac{1}{\operatorname{rank} \bar{M}_{d}} \cdot\left(1-\frac{\operatorname{rank} \bar{K}_{d}}{\operatorname{rank} F_{d}}\right),
$$

Thus,

$$
\frac{\operatorname{rank} \bar{M}_{d}}{\operatorname{rank} \bar{M}_{d}+h^{0}(B)}>1-\frac{\operatorname{rank} \bar{K}_{d}}{\operatorname{rank} F_{d}}
$$

$$
\frac{\operatorname{rank} \bar{K}_{d}}{\operatorname{rank} F_{d}}>1-\frac{\operatorname{rank} \bar{M}_{d}}{\operatorname{rank} \bar{M}_{d}+h^{0}(B)}=\frac{h^{0}(B)}{\operatorname{rank} M_{d}},
$$


and hence

$$
\operatorname{rank} \bar{K}_{d}>h^{0}(B) \cdot\left(\frac{\operatorname{rank} F_{d}}{\operatorname{rank} M_{d}}\right) .
$$

However, by the previous lemma, rank $F_{d} \geq h^{0}\left(X, L_{d}-B\right)-1$. Furthermore, $B$ is independent of $d$, and rank $M_{d}=h^{0}\left(X, L_{d}\right)-1$. Thus as $d$ grows, the fraction on the right in $\left(^{*}\right)$ becomes arbitrarily close to $1 .{ }^{1}$ It follows that

$$
\operatorname{rank} \bar{K}_{d}>h^{0}(B)-1
$$

provided that $d \gg 0$, and hence rank $\bar{K}_{d}=h^{0}(B)$, as required.

\section{Complements}

In this section, we first of all prove Proposition $\mathrm{C}$ by adapting the method of proof of Theorem 1.1 in [6]. Then we propose some open problems.

2.1. Coandă's argument. We begin by stating (without proof) two preliminary results on which the method rests; the first of these is a cohomological characterization of stability, and the second is a vanishing theorem of Green.

Lemma 2.1. Let $E$ be a vector bundle on $X$. If for every $r$ with $0<r<\operatorname{rk}(E)$ and for every line bundle $N$ on $X$ with $\mu_{L}\left(\Lambda^{r} E \otimes N\right) \leq 0$ one has $H^{0}\left(\Lambda^{r} E \otimes N\right)=0$, then $E$ is $L$-stable.

Lemma 2.2 ([10, 3.a.1]). Let $N, N^{\prime}$ be line bundles on $X$ and assume $N$ is very ample. Then for $r \geq h^{0}\left(N^{\prime}\right)$, we have $H^{0}\left(\Lambda^{r} M_{N} \otimes N^{\prime}\right)=0$.

Proof of Proposition $C$. Let $X$ be a smooth projective variety of dimension $n \geq 2$ for which $\operatorname{Pic}(X) \cong \mathbb{Z} \cdot[A]$ for an ample line bundle $A$. Consider the function $q: \mathbb{N} \rightarrow \mathbb{Q}$ defined by $q(t)=\frac{h^{0}(t A)-1}{t}$. Since $q(t)=\frac{A^{n}}{n !} t^{n-1}+O\left(t^{n-2}\right)$ for $t \gg 0$ by asymptotic Riemann-Roch, there exists a positive integer $d_{0}$ satisfying the following properties:

(1) For all integers $a$ satisfying $1 \leq a \leq d_{0}-1$, we have $q(a)<q\left(d_{0}\right)$.

(2) For all integers $d \geq d_{0}$, we have that $d A$ is very ample and $q(d)<q(d+1)$.

An immediate consequence is that $q(a)<q(d)$ whenever $d \geq d_{0}$ and $1 \leq a \leq d-1$. For the rest of the proof, we fix an integer $d \geq d_{0}$.

Recalling that $\operatorname{Pic}(X)=\mathbf{Z} \cdot[A]$ by assumption, it suffices by Lemmas 2.1 and 2.2 to show that given integers $a$ and $0<r<h^{0}(d A)-1$, one has the implication

$$
\mu_{A}\left(\Lambda^{r} M_{d} \otimes \mathcal{O}_{X}(a A)\right) \leq 0 \Longrightarrow r \geq h^{0}(a A),
$$

where as before $M_{d}=M_{d A}$. This is automatic for $a \leq 0$, so we assume $a \geq 1$ throughout. We have that

$$
\mu_{A}\left(\Lambda^{r} M_{d} \otimes \mathcal{O}_{X}(a A)\right)=r \cdot \mu_{A}\left(M_{d}\right)+a \cdot\left(A^{n}\right)=\left(A^{n}\right) \cdot\left(a-\frac{d r}{h^{0}(d A)-1}\right)
$$

Our assumption that $\mu_{A}\left(\Lambda^{r} M_{d} \otimes \mathcal{O}_{X}(a A)\right) \leq 0$ then implies that $a \leq \frac{d \cdot r}{h^{0}(d A)-1}$, or

$$
r \geq a \cdot\left(\frac{h^{0}(d A)-1}{d}\right) .
$$

\footnotetext{
${ }^{1}$ In fact, $h^{0}\left(L_{d}\right)-h^{0}\left(L_{d}-B\right)=O(d)$, whereas $h^{0}\left(L_{d}\right)$ grows quadratically in $d$.
} 
In particular, $a<d$, so $1 \leq a \leq d-1$. We will be done once we verify that

$$
a \cdot\left(\frac{h^{0}(d A)-1}{d}\right)>h^{0}(a A)-1,
$$

for $1 \leq a \leq d-1$. However, (2.4) is equivalent to $q(a)<q(d)$, so this follows from our assumption on $d$.

Remark 2.3 (Rigidity of $\boldsymbol{M}_{\boldsymbol{L}}$ ). Let $L$ be a very ample line bundle on a smooth complex projective variety $X$ of dimension $\geq 3$ with $H^{1}\left(X, \mathcal{O}_{X}\right)=0$. Then arguing as in the proof of [5, Proposition 1], one sees that $M_{L}$ is rigid, i.e., $\operatorname{Ext}^{1}\left(M_{L}, M_{L}\right)=0$. Consequently, in the situation of Proposition C, $M_{d}$ again represents an isolated point of the moduli space of bundles when $\operatorname{dim}_{\mathbf{C}} X \geq 3$ and $d \gg 0$.

2.2. Some open problems. Recall that if $X$ is a smooth curve of genus $g \geq 1$, then $M_{L}$ is stable as soon as $\operatorname{deg} L \geq 2 g+1$. This suggests

Problem 2.4. Find an effective version of Theorem A.

Presumably one would want to work with divisors of the sort $L=K_{X}+B+N$ with $B$ satisfying a suitable positivity hypothesis, and $N$ nef.

It is also interesting to ask whether $M_{d}$ satisfies some stronger stability properties:

Problem 2.5. As before, let $L_{d}=d A+P$, and put $M_{d}=M_{L_{d}}$. Is $M_{d}$ slope stable with respect to any polarization on $X$ when $d \gg 0$ ? In characteristic $p>0$, is it strongly stable?

Finally, we conjecture that our main result extends to all dimensions.

Conjecture 2.6. Let $X$ be a smooth projective variety of dimension $n$, and define $M_{d}$ as above. Then $M_{d}$ is $A$-stable for every $d \gg 0$.

\section{Acknowledgments}

Research of Lawrence Ein partially supported by NSF grant DMS-1001336. Research of Robert Lazarsfeld partially supported by NSF grant DMS-1159553. Research of the Yusuf Mustopa partially supported by NSF grant RTG DMS-0502170. We are grateful to M. Mustaţă and V. Srinivas for valuable discussions, and to the referee for some useful suggestions.

\section{References}

[1] A. Beauville, Some stable vector bundles with reducible theta divisor, Manuscr. Math. 110 (2003), 343-349.

[2] H. Brenner, Looking out for stable syzygy bundles, with an appendix by Georg Hein, Adv. Math. $\mathbf{2 1 9}(2)$ (2008), 401-427.

[3] D. Butler, Normal generation of vector bundles over a curve, J. Differ. Geom. 39 (1994), 1-34.

[4] C. Camere, About the stability of the tangent bundle of $\mathbf{P}^{n}$ restricted to a curve, C. R. Acad. Sci. Paris, Ser. I 346 (2008), 421-426.

[5] C. Camere, About the stability of the tangent bundle of $\mathbb{P}^{n}$ restricted to a surface, Math. Zeit. 271(1-2) (2012), 499-507.

[6] I. Coandă, On the stability of syzygy bundles, Int. J. Math. 22(4) (2011), 515-534.

[7] L. Costa, P. Macias Marques and R.M. Miró-Roig, Stability and unobstructedness of syzygy bundles, J. Pure Appl. Algebr. 214(7) (2010), 1241-1262. 
[8] L. Ein and R. Lazarsfeld, Stability and restrictions of Picard bundles, with an application to the normal bundles of elliptic curves, in 'Complex Projective Geometry', London Math. Soc. Lecture Note Ser. 179 (1992), 149-156.

[9] H. Flenner, Restrictions of semistable bundles on projective varieties, Comment. Math. Helv. 59 (1984), 635-650.

[10] M. Green, Koszul cohomology and the geometry of projective varieties, J. Differ. Geom. 19 (1984), 125-171.

[11] P. Macias Marques and R.M. Miró-Roig, Stability of syzygy bundles, Proc. Amer. Math. Soc. 139 (2011), 3155-3170.

[12] E. Mistretta, Stability of line bundle transforms on curves with respect to low codimensional subspaces, J. Lond. Math. Soc. (2) 78 (2008), 172-182.

[13] K. Paranjape and S. Ramanan, On the canonical ring of a curve, in 'Algebraic Geometry and Commutative Algebra', vol. II, Kinokuniya, Tokyo (1988) 503-516.

[14] V. Trivedi, Semistability of syzygy bundles on projective spaces in positive characteristics, Int. J. Math. 21(11) (2010), 1475-1504.

Department of Mathematics, University Illinois at Chicago, 851 South Morgan St., Chicago, IL 60607, USA

E-mail address: ein@uic.edu

Department of Mathematics, University of Michigan, Ann Arbor, Mi 48109, USA

E-mail address: rlaz@umich.edu

Department of Mathematics, Boston College, Chestnut Hill, MA 02467, USA

E-mail address: mustopa@bc.edu 